\section{L'Actualité économique}

L'ACTUALITÉ ÉCONOMIQUE

The Nature and Efficiency of the Foreign Exchange Market, par JEROME-L. STEIN. Une brochure, 6 po. $x$ 9, 58 pages - ESSAYS IN INTERNATIONAL FINANCE, NO. 40, International Finance Section, Princeton University, 1962

\title{
Bernard Bonin
}

Volume 39, numéro 1, avril-juin 1963

URI : https://id.erudit.org/iderudit/1001896ar

DOI : https://doi.org/10.7202/1001896ar

Aller au sommaire du numéro

Éditeur(s)

HEC Montréal

ISSN

0001-771X (imprimé)

1710-3991 (numérique)

Découvrir la revue

Citer ce compte rendu

Bonin, B. (1963). Compte rendu de [The Nature and Efficiency of the Foreign Exchange Market, par JEROME-L. STEIN. Une brochure, 6 po. x 9, 58 pages ESSAYS IN INTERNATIONAL FINANCE, NO. 40, International Finance Section, Princeton University, 1962]. L'Actualité économique, 39(1), 143-144.

https://doi.org/10.7202/1001896ar d'utilisation que vous pouvez consulter en ligne. 


\section{LES LIVRES}

que valeur symbolique, illustrant la possibilité pour tous de devenir chef d'entreprise et en sousentendu d'aspirer à la richesse.

Le petit homme d'affaires est beaucoup plus sensible que son confrère de la grande industrie aux fluctuations économiques. Son meilleur moyen de défense semble être le fait d'appartenir à une association qui, elle, pourra avoir à son service des spécialistes en économie, en marketing, en relations industrielles. Ces spécialistes pourront donner aux membres des directives leur permettant de mieux faire face aux événements.

Un point à retenir à propos de ces associations : c'est que la majorité d'entre elles ressemblent beaucoup aux entreprises qu'elles représentent, en ce sens qu'elles sont la plupart du temps l'affaire d'un seul homme, le fondateur, qui la dirige avec la main de fer du petit entrepreneur.

En conclusion, ce livre a certainement une valeur documentaire pour le chercheur qui s'intéresse à la petite entreprise américaine. Même si la majeure partie de l'ouvrage traite d'associations américaines, certains chapitres (le rer et le zème) examinent des points généraux assez intéressants même pour le lecteur canadien.

Paul Dell'Aniello

The Nature and Efficiency of the Foreign Exchange Market, par Jerome-L. Stein. Une brochure, 6 po. $x$ 9, 58 pages. - Essays IN InTERNATIONAL FinANCE, NO. 40, International Finance Section, Princeton University, 1962.

L'auteur est professeur d'économie politique à Brown University. La brochure qu'il nous présente est extrêmement intéressante à plusieurs points de vue. Elle constitue tout d'abord une remarquable étude du fonctionnement du marché des changes. De plus, dans un appendice, l'auteur examine le taux de change entre le sterling et le dollar durant la période $1921-1925$, c'est-à-dire durant une période de libre fluctuation selon les forces du marché dans le but de déterminer dans quelle mesure le taux de change futur pouvait être prévu. Mais la brochure contient également une analyse remarquable de la technique des opérations de change qui lui confère un intérêt particulier. Elle devient ainsi un document fondamental non seulement pour un étudiant en économie politique, mais aussi pour un étudiant qui se destine à la finance.

Dans une première partie, l'auteur étudie les fonctions du marché des changes. Il s'intéresse spécialement, dans la deuxième partie, à l'activité des grandes banques (professional risk-Bearers) et des placeurs à court terme sur le marché des changes. Le lecteur trouvera, dans cette partie, une masse de renseignements extrêmement intéressants sur la gestion d'un portefeuille de devises. Enfin, dans une troisième partie, il étudie l'ajustement du marché des changes dans une période \& normale » et dans une période marquée par de fortes spéculations. Encore là, on trouvera des renseignements fort utiles sur le fonctionnement du marché instantané (spot market) et du marché à terme (forward market). Il applique ensuite sa théorie à la réévalution du mark (196r) et à la course sur 


\section{L'ACTUALITÉ ECONOMIQUE}

le sterling (juin 1961). Les partisans du taux de change fluctuant trouveront, dans l'appendice, des arguments à l'appui de leur thèse. Bernard Bonin

Labour Arbitration in Canada, par A.-W.-R. CARRothers. Un vol., $61 / 2$ po. x 91/2, relié, 204 pages. - BUTTERWORTHS, Toronto, 1961.

Monsieur A.W.R. Carrothers, professeur de Droit et directeur de l'Institut des Relations industrielles de l'Université de la Colombie-Britannique, analyse dans un ouvrage, les différentes procédures d'arbitrage qui existent dans chacune des provinces canadiennes de droit commun.

Il essaie de montrer qu'en même temps qu'elles accordent aux ouvriers le droit de s'associer en vue de revendiquer pour améliorer leurs salaires et leurs conditions de travail, les différentes législations ouvrières de ces provinces doivent prévoir, du même coup, un mécanisme de réglementation des disputes qui s'élèvent entre patrons et syndiqués.

Parmi les deux grandes catégories de conflits qui surviennent au sein de l'entreprise syndiquée (les conflits d'ordre économique, survenant lors de la négociation de la convention collective, et ceux qui concernent la violation de droits établis au préalable par les deux parties et qui prennent naissance au cours de l'administration de cette même convention) l'auteur ne retient que la deuxième.

Après un court exposé sur l'histoire de la législation ouvrière canadienne, M. Carrothers analyse la loi des syndicats ouvriers (trade union act) des provinces anglaises afin de vérifier si le droit d'association est bien reconnu aux travailleurs de ces mêmes provinces. Une fois que le statut légal de la convention collective est reconnu, il s'applique à vérifier, par l'examen des différentes lois provinciales sur les relations ouvrières (labour relations act), si un mécanisme de réglementation des griefs a également été prévu.

L'auteur consacre la majeure partie de son volume à l'analyse de ce double mécanisme : le conseil de conciliation et le conseil d'arbitrage. Après avoir décrit la fonction du conseil de conciliation, sa composition, son objectif, il concentre son étude sur la façon dont est constitué le tribunal d'arbitrage, une fois que le conseil de conciliation s'est révélé impuissant à régler la dispute, sur les pouvoirs que les différentes lois provinciales lui accordent. Il étudie enfin la loi sur l'arbitrage (arbitration act) des provinces anglaises en vue d'y découvrir les différentes procédures d'arbitrage qui sont en vigueur et d'y dégager la valeur juridique du jugement final que les membres du tribunal doivent rendre après avoir siégé.

L'auteur alimente son analyse de la législation ouvrière des provinces anglaises, de nombreux exemples pratiques puisés dans les décisions prises au cours de différentes disputes.

Cette étude très sérieuse du mécanisme de réglementation des griefs, tel qu'il existe au Canada anglais, mérite notre attention. Elle représente une bonne synthèse. L'auteur a su regrouper et agencer, sur une base comparative, ces nombreux articles de lois. 